\title{
Plasmocitoma extramedular de laringe. A propósito de un caso
}

\section{Extramedullary plasmacytoma of the larynx. A case report}

\author{
Constanza Bulboa F. ${ }^{1}$, Gerard Pujals T. ${ }^{1}$, Ana Holgado C. ${ }^{1}$, Albert Llansana R. ${ }^{1}$
}

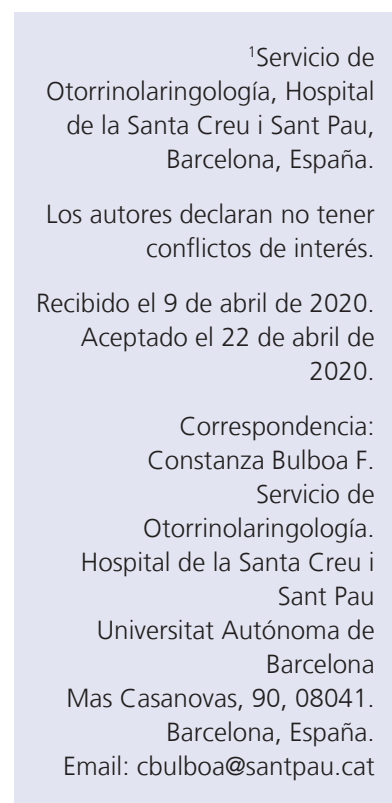

\section{Resumen}

Los plasmocitomas solitarios son una rara forma de presentación de neoplasias de células plasmáticas. Deben ser diferenciados del mieloma múltiple en el momento del diagnóstico y realizar un seguimiento estricto por el riesgo de transformación a mieloma. La localización de los mismos en la laringe es muy inusual, dado que sólo se han publicado 56 casos. Se presenta el caso de una paciente con un plasmocitoma extramedular de laringe que se trató mediante cirugía y radioterapia. Se revisan los criterios diagnósticos y los problemas terapéuticos.

Palabras clave: plasmocitoma solitario, plasmocitoma extramedular, mieloma múltiple.

\begin{abstract}
Solitary plasmacytomas are a rare form of plasma cell neoplasms. They should be differentiated from multiple myeloma at the time of diagnosis and strictly monitored for the risk of transformation to myeloma. Their location in the larynx is very unusual, since only 56 cases have been published. We present the case of a patient with extramedullary plasmacytoma of the larynx that has been treated with surgery and radiotherapy. We reviewed the literature for diagnostic criteria and therapeutic problems.

Keywords: solitary plasmacytoma, extramedullary plasmacytoma, multiple myeloma.
\end{abstract}

\section{Introducción}

Los plasmocitomas solitarios (PS) son una proliferación de células plasmáticas aisladas cuyo origen radica en el sistema reticuloendotelial ${ }^{1}$. Estos tumores son infrecuentes y afectan con mayor preponderancia a varones de más de 50 años. Los principales síntomas están relacionados con el crecimiento local ${ }^{2}$. Los PS se clasifican en: plasmocitoma óseo solitario (POS) y plasmocitoma extramedular (PEM). El POS afecta preferentemente huesos largos y esqueleto axial, siendo infrecuente a nivel de cabeza y cuello ${ }^{1,2}$. En contraste los PEM se ubican en áreas submucosas y un $80 \%-90 \%$ se presentan a nivel de cabeza y cuello, específicamente a nivel del tracto aerodigestivo superior, preferentemente a nivel nasosinusal. Por lo general, las lesiones son únicas e independiente y un $10 \%-30 \%$ puede progresar a mieloma múltiple $(\mathrm{MM})^{1}$.

El PEM laríngeo es especialmente infrecuente y representa el $10 \%$ del total de plasmocitomas y entre 0,04\%-0,19\% del total de neoplasias malignas de la laringe ${ }^{2}$. En la laringe la zona más afectada es la supraglotis ${ }^{3}$. Los PEM de cabeza y cuello se presentan mayormente en forma localizada, siendo poco frecuente que presenten adenopatías en el momento del diagnóstico (5\%-20\% de los casos $)^{4}$. El diagnóstico es histológico e inmunohistoquímico, donde se observan células plasmáticas monoclonales ${ }^{2}$. En el estudio hematológico, se debe solicitar un hemograma completo, electroforesis en suero/orina, cuantificación de inmunoglobulinas (Ig), biopsia de médula 
ósea y estudio de extensión ${ }^{4}$. La radioterapia se considera la piedra angular del tratamiento. La cirugía también se ha empleado como tratamiento en un porcentaje variable de pacientes ${ }^{5}$. En este artículo presentamos el caso de una paciente con plasmocitoma extramedular a nivel laríngeo, y se revisan las características de esta patología.

\section{Caso Clínico}

Mujer de 52 años, que consultó por cuadro de 3 años de evolución de aumento de su roncopatía, hace 4 meses se agregó disnea de esfuerzo moderado y, posteriormente, disfonía. Niega disfagia, baja de peso o fiebre. Como antecedentes personales destacó lupus eritematoso sistémico, síndrome de Sjögren y enfermedad pulmonar quística secundaria a neumonía intersticial linfocítica. En la exploración, se observó lesión submucosa en repliegue ariteno-epiglótico derecho con extensión hacia banda ventricular ipsilateral, sin provocar alteración en la movilidad de cuerdas vocales (Figura 1), y en el examen cervical no se palparon adenopatías.

A la tomografía computarizada (TC) se observó una lesión hipervascularizada a nivel de la mucosa del repliegue ariteno-epiglótico hasta la banda y el ventrículo derecho, por lo que se sospechó un paraganglioma laríngeo (Figura 2).

Se complementó estudio con resonancia magnética (RM) que fue concordante con la lesión tipo paraganglioma (lesión redondeadas bien delimitadas, isodensas respecto del músculo, con importante reforzamiento postcontraste intravenoso). Debido a la sospecha de paraganglioma laríngeo se solicita estudio de rastreo gamagráfico con somatostatina donde se observó marcación a nivel laríngeo y se descartó multifocalidad de la patología. No se realizó biopsia previa a la intervención quirúrgica por el riesgo elevado de sangrado. Se decidió realizar angiografía y embolización de las ramas de arteria tiroidea superior derecha y, posteriormente, se realizó un abordaje externo lateral de la laringe a nivel supraglótica derecho. Después de seccionar la musculatura infrahioidea, se expuso el ala derecha del cartílago tiroides y se procedió a resecar su tercio superior incluyendo el asta superior para tener un buen acceso al espacio paraglótico superior y al espacio preepiglótico derecho. En esa región se encontró el tumor que se fue disecando cuidadosamente hasta su exéresis completa (Figura 3), además, se realizó conjuntamente una traqueostomía de seguridad. Evolucionó sin incidentes en su postoperatorio.

A la anatomía patológica se informó un tumor de células plasmáticas con márgenes

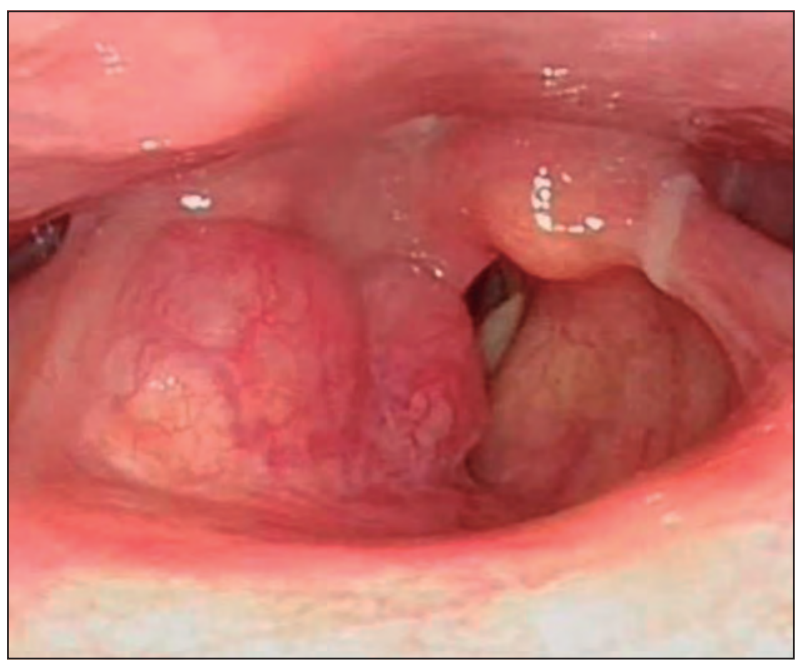

Figura 1. Imagen de fibroscopio de lesión submucosa en supraglotis derecha.

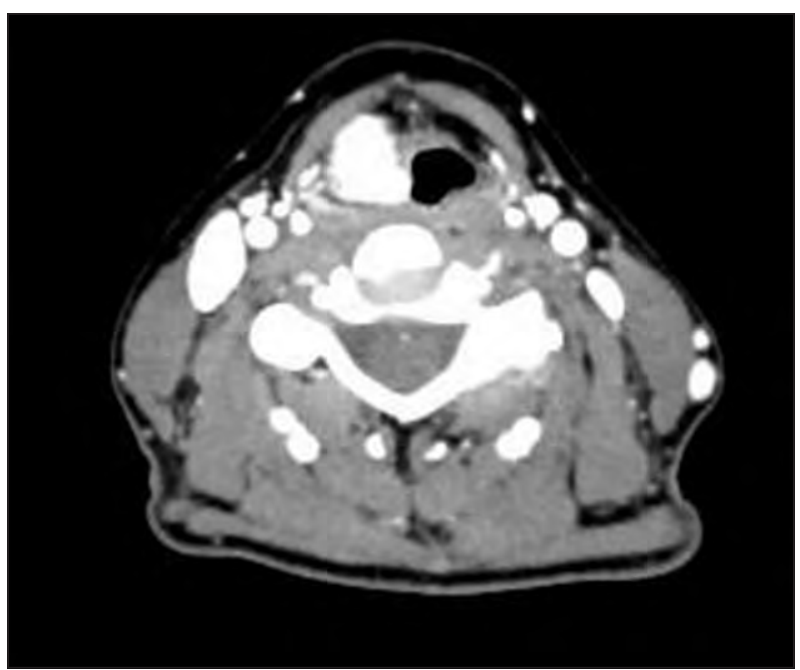

Figura 2. Tomografía computada de cuello, corte axial. Se observa lesión hipervascularizada a nivel repliegue aritenoepiglótico derecho. 


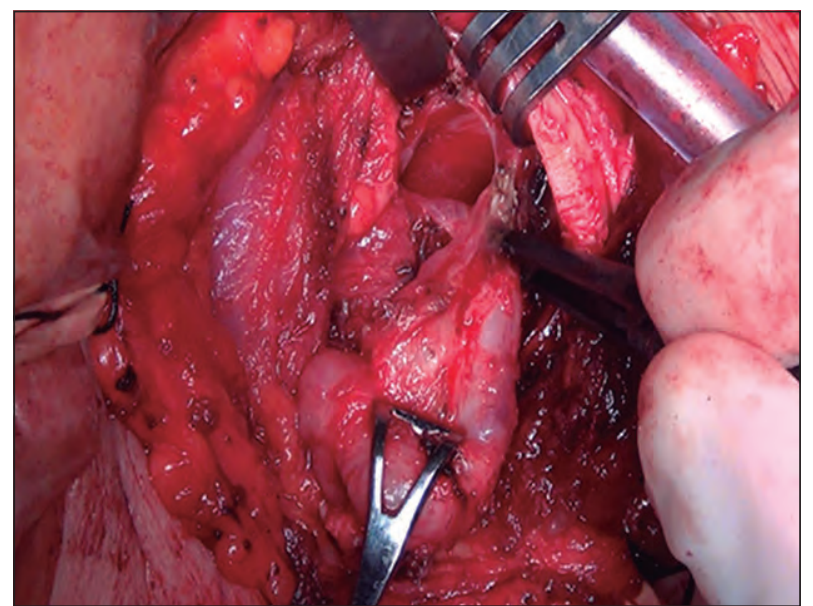

Figura 3. Exéresis de lesión submucosa de espacio paraglótico y preepiglótico derecho.

afectos. Las tinciones inmunohistoquímicas mostraron que la celularidad neoplásica era positiva para $\mathrm{CD}^{1,3,8}$ y cadena ligera kappa. Las tinciones para cromogranina, sinaptofisina, queratina AE1/AE3 fueron negativas. Se derivó para continuar estudio con equipo de hematología, quienes solicitaron analítica sanguínea con hemograma completo, bioquímica, cuantificación de inmunoglobulinas, inmunoelectroforesis en orina y sangre, los cuales se encontraron dentro de los límites de la normalidad (no había un peak monoclonal). La radiografía de tórax y PET-TC, en busca de lesiones osteolíticas o fracturas patológicas, fueron normales. Además, se solicitó aspirado de médula ósea y biopsia que descartan mieloma múltiple. Se inicia tratamiento con radioterapia a dosis de $40 \mathrm{~Gy}$ fraccionado. Al año del diagnóstico se encuentra en remisión completa y con correcta función laríngea.

\section{Discusión}

Los PS son tumores infrecuentes con una incidencia de $3 / 100.000$ personas/año, representando menos del $1 \%$ de tumores de cabeza/ cuello $^{2}$. El PEM es un subtipo de PS, que se origina en tejidos blandos y es más frecuente en cabeza/cuello ${ }^{1}$. En la revisión bibliográfica de Alexious y cols. donde se revisaron más de 400 publicaciones sobre PEM, se constató que cabeza y cuello es la primera localización de estos tumores ${ }^{6}$. Dentro de cabeza y cuello, la laringe es una localización poco habitual, y en nuestra revisión de la literatura sólo hemos encontrado 56 casos de PEM laríngeo.

El síntoma más frecuente de PEM de ubicación laríngea suelen ser disfonía, disfagia y disnea. La presencia de adenopatías es variable de $0 \%-25 \%{ }^{7}$. En el caso de nuestra paciente el síntoma inicial fue la roncopatía que corresponde a un síntoma inusual que no se describe con frecuencia en la literatura, pero que se explica por el efecto masa del tumor submucoso.

La etiología de los PEM es desconocida; se ha relacionado con el virus de Epstein-Barr y la irritación crónica por agentes inhalados ${ }^{2}$. El diagnóstico diferencial de una tumoración submucosa laríngea, debe incluir neoplasias malignas como el carcinoma escamoso u otras histologías malignas, pero también lesiones benignas. El primer diagnóstico sospechado en nuestro caso fue de paraganglioma por la edad de la paciente, lentitud en su evolución y, sobre todo, porque en el TC se presentaba como un tumor muy vascularizado ${ }^{8}$. El diagnóstico del PS es histológico, se establece por presencia de células plasmáticas con atipias. La naturaleza monoclonal de las células se confirma por inmunohistoquímica ${ }^{7}$.

Para poder confirmar el diagnóstico de PEM se deben cumplir los siguientes criterios: (I) lesión tumoral compatible con plasmocitoma en la histología; (II) ausencia de células plasmáticas en médula ósea (confirmada por biopsia); (III) ausencia de anemia/hipercalcemia o insuficiencia renal; (IV) niveles normales de inmunoglobulinas policlonales $y$ ausencia de componente monoclonal en sangre $y / u$ orina 9 . Dentro del estudio se debe realizar PET/TC corporal o resonancia magnética para excluir la presencia de otras lesiones malignas. El grupo de trabajo internacional de mieloma, recomienda PET/ TC como parte de la evaluación inicial de pacientes con PEM con el fin de detectar otras lesiones adicionales 9 . El PEM presenta un crecimiento esencialmente local a diferencia del subtipo plasmocitoma óseo solitario, cuya progresión a MM es mayor ${ }^{5}$.

En relación con el tratamiento, las publicaciones muestran combinaciones y resultados dispares. Bachar y cols. recomiendan como mejor tratamiento la radioterapia, reservan- 
do la cirugía para las lesiones extensas o con compromiso óseo ${ }^{4}$. Venkatesulu y cols., en una revisión de estudios de PEM en cabeza/cuello, concluyeron que la combinación cirugía y radioterapia es superior a las terapias unimodales dado que cuando se realiza cirugía más radioterapia hay menos recidivas locales 5 . Alexiou y cols. a raíz de una revisión bibliográfica de los casos de PEM, concluyeron que la cirugía sola da mejores resultados que las otras técnicas, siempre que la resección haya sido adecuada ${ }^{6}$. En la última revisión realizada por expertos hematólogos europeos se señaló a la radioterapia como el mejor tratamiento. La dosis recomendada fue de 40-50 Gy fraccionados, reservando la cirugía para tumoraciones grandes y bien definidas, aunque se sugieren añadir radioterapia postoperatoria9. Los factores de riesgo de recidiva local que se han descrito son la ubicación nasosinusal y haber recibido quimioterapia unimodal ${ }^{5}$. El seguimiento se aconseja que sea de por vida dado que la recidiva puede desarrollarse muchos años después ${ }^{4}$. Con respecto a la quimioterapia adyuvante los datos publicados son poco concluyentes. En el foro de mieloma del Reino Unido, se sugirió que la quimioterapia se considere en PEM mayores de $5 \mathrm{~cm}$, tumores de alto grado y enfermedad refractaria/recidivante ${ }^{10}$.

\section{Conclusión}

Los PS son tumores infrecuentes de cabeza/ cuello y la mayoría corresponde a plasmocitoma extramedular de ubicación nasosinusal. La localización a nivel laríngeo es muy inusual ${ }^{2}$. En nuestra revisión, sólo se han encontrado 56 casos reportados como PEM a nivel laríngeo. Dentro del diagnóstico, es de suma importancia la exclusión de $\mathrm{MM}^{1}$. La radiación se considera la piedra angular del tratamiento, y la cirugía también se ha empleado como tratamiento curativo en un porcentaje de los pacientes $^{5}$.

\section{Bibliografía}

1. Kilciksiz S, Karakoyun-Celik O, Agaoglu FY, Haydaroglu A. A review for solitary plasmacytoma of bone and extramedullary plasmacytoma. Scientific World Journal 2012; 2012:895765. Disponible en https://doi.org/10.1100/2012/895765.

2. Ramírez-Anguiano J, Lara-Sánchez H, MartínezBaños D, Martínez-Benítez B. Extramedullary Plasmacytoma of the Larynx: A Case Report of Subglottic Localization. Case Rep Otolaryngol 2012;2012:1-3.

3. Soni NK, Trivedi KA, Kumar A, et al. Solitary extramedullary plasmacytoma - Larynx. Indian J Otolaryngol Head Neck Surg 2002;54:309-10.

4. Bachar G, Goldstein D, Brown D, et al. Solitary extramedullary plasmacytoma of the head and necklong-term outcome analysis of 68 cases. Head Neck 2008; 30:1012-1019. doi:10.1002/hed.20821.

5. Venkatesulu B, Mallick S, Giridhar P, Upadhyay $A D$, Rath GK. Pattern of care and impact of prognostic factors on the outcome of head and neck extramedullary plasmacytoma: a systematic review and individual patient data analysis of 315 cases. Eur Arch Otorhinolaryngol 2018;275:595-606. Disponible en https://doi.org/10.1007/s00405-017-4817-z.

6. Alexiou C, Kau RJ, Dietzfelbinger H, et al. Extramedullary plasmacytoma: tumor occurrence and therapeutic concepts. Cancer 1999; 85:2305-14.

7. Fernández Pérez AJ, Sancho Mestre M, Gras Albert JR, Talavera Sánchez J. Plasmocitoma solitario de cabeza y cuello. Presentación de tres casos y revisión de la literatura. Acta Otorrinolaringol Esp 2001; 52:715-20.

8. Higuera A, Palomo V, Muñoz R, Sánchez F. Neurinoma laríngeo. Diagnóstico diferencial de tumoraciones submucosas laríngeas. Radiologia 2002; 44:170-2. Disponible en https://doi.org/10.1016/ S0033-8338(02)77785-0.

9. Caers J, Paiva B, Zamagni E, et al. Diagnosis, treatment, and response assessment in solitary plasmacytoma: Updated recommendations from a European Expert Panel. J Hematol Oncol 2018;11:110.

10. Strojan P, Šoba E, Lamovec J, Munda A. Extramedullary plasmacytoma: Clinical and histopathologic study. Int J Radiat Oncol Biol Phys 2002; 53:692-701. 